\title{
A low drying temperature benefits the quality of dehydrated thyme
}

\author{
Chao Zhang ${ }^{\mathrm{a}}$, Jiangchangmei Lu ${ }^{\mathrm{b}}$, Yubin $\mathrm{Wang}^{\mathrm{c}}$, Yue $\mathrm{Ma}^{\mathrm{d}}$, Xiaoyan Zhao ${ }^{\mathrm{e}}$
}

Beijing Vegetable Research Center, Beijing Academy of Agriculture and Forestry Sciences; Beijing

Key Laboratory of Fruits and Vegetable Storage and Processing; Key Laboratory of Biology and Genetic Improvement of Horticultural Crops (North China), Ministry of Agriculture; Key Laboratory of

Urban Agriculture (North), Ministry of Agriculture, 9 Shuguanghuayuan Road, Haidian District, Beijing, China

azhangchao@nercv.org, 'blujiangchangmei@nercv.org, 'wangyubin@nercv.org, 'mayue@nercv.org, ezhaoxiaoyan@nercv.org

Keywords: thyme, dehydration, drying temperature, water activity

Abstract. The effect of drying temperature on qualities of dehydrated thyme was evaluated. The moisture content and water activity of the thyme decreased with the drying time and drying temperature. The chlorophyll content was enhanced when the drying temperature was decreased. Hence, a lower drying temperature benefited to hold the quality of dehydrated thyme.

\section{Introduction}

Thyme (Thymus vulgaris L.) belongs to the Lamiaceae family and consists of about 215 species spread worldwide [1]. The Mediterranean region is considered as the center of origin of that genus [2]. Fresh leave and flowers of thyme can be used as aroma additive in food, pharmaceuticals and cosmetics [3].

Thyme is perishable due to its high moisture content $(65 \% \sim 68 \%)$, thereby requiring the use of preservation methods for its further use [4]. Hot air drying is one of the traditional food preservation method for most high moisture materials. Dehydration extends product shelf life, minimizes packaging requirements and reduces shipping weights $[4,5]$. The dehydration increases the shelf life by slowing microorganisms growth and preventing certain biochemical reactions that might alter the organoleptic characteristics $[5,6]$. Thyme has a pleasant smell and taste due to its essential oil [7]. Therefore, the thyme was dehydrated at a different temperature $\left(45 \sim 85^{\circ} \mathrm{C}\right)$. The qualities of the dehydrated thyme were compared.

\section{Material and Methods}

Dehydration of thyme. Thyme was picked from our Tongzhou farm (Tongzhou District Beijing, 2014). The fresh leaf of the thyme was stored at $4{ }^{\circ} \mathrm{C}$ before use. The thyme was washed by the tap water at $4{ }^{\circ} \mathrm{C}$ to remove the soil and some inclusion. The washed thyme was heated at $45,55,65,75$, and $85{ }^{\circ} \mathrm{C}$ in a air-circulated oven respectively. The moisture content of $7 \%$ was the termination for each treatment. During each treatment, about 7 samples were picked up for the quality determination. The fresh thyme was designated as the control.

Determination of chlorophyll by spectrophotometry. To measure the chlorophyll content, $20 \mathrm{ml}$ of $80 \%$ acetone was added to $5 \mathrm{~g}$ of each sample and mixed with a homogenizer (IKA T10 Basic, Werke GmbH \& Co. KG, Staufen, Germany) for $1 \mathrm{~min}$ at a high speed, then centrifuged at 8,000 rpm for $5 \mathrm{~min}$ at $4{ }^{\circ} \mathrm{C}$, and then filtered through Whatman No. 1 filter papers. The volume was adjusted to $25 \mathrm{~mL}$ and the absorbance values were measured at 663 and $645 \mathrm{~nm}$ by a 
spectrophotometer (UV-1800, Shimadzu Corporation, Kyoto, Japan). The chlorophyll content of the sample was calculated followed by Equation 1 .

$$
\text { Chlorophyll }=\frac{\left(20.2 \times A_{645}+8.02 \times A_{663}\right) \times D}{1000}
$$

where chlorophyll content is $\mathrm{mg} / \mathrm{g}$ fresh weight; $A_{645}$ and $A_{663}$ are the absorbance at $645 \mathrm{~nm}$ and $663 \mathrm{~nm}$, respectively; $D$ is the dilution factor of the sample.

Moisture content determination. The moisture content of the sample was measured by heating at $105{ }^{\circ} \mathrm{C}$ until a constant weight was reached. The moisture content was the percentage of the weight loss in the total weight.

Water activity determination. The water activity of the sample was measured by a Aqua LAB 4TE (Decagon Devices, USA). The Aqua LAB 4TE was calibrated by the water activity standard solution of $1.0,0.75$ and 0.5 respectively before measurement. The sample was powdered and was covered on the sample plate. The data was recorded when the result was constant.

Statistical Analysis. Analysis of variance (ANOVA) was used to compare mean differences of the results. If the differences in mean existed, multiple comparisons were performed using Duncan's Multiple Range Test. All analysis was conducted using SPSS for Window Version 19. All experiments were done in triplicates or more.

\section{Results and Discussion}

Effect of dehydration on moisture content of thyme. The effect of dehydration on the moisture content of the thyme is shown in Figure 1.The moisture content decreased with the drying time. The drying processing was able to be divided into 3 stages. Firstly, the moisture content decreased slowly, and then decreased quickly. Finally, the moisture content reached to a constant level of $2 \% \sim 7 \%$. The drying time was depended on the drying temperature. A higher drying temperature reduced the drying time. For instants, the drying temperature of $45{ }^{\circ} \mathrm{C}$ cost $260 \mathrm{~min}$ to reach the moisture content of $7 \%$, while drying temperature of $95{ }^{\circ} \mathrm{C}$ only cost $65 \mathrm{~min}$. Similar results also presented in the dehydration of cabbage [8], carrot [9], and mint [10].

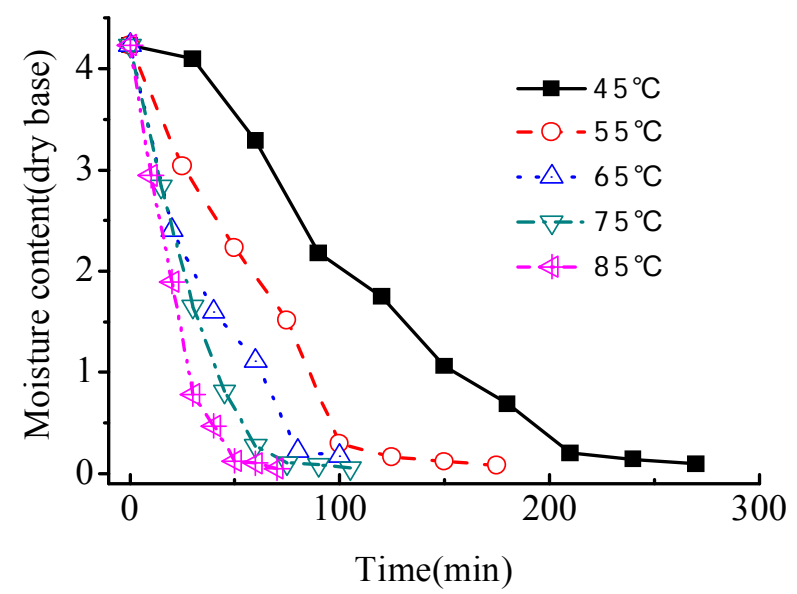

Figure 1 Effect of dehydration on moisture content of thyme

Effect of dehydration on water activity of thyme. Water activity is an important consideration for the sensory properties and safety of foods, which is defined as the vapor pressure of a liquid divided by that of pure water at the same temperature [11]. Nearly no microorganisms survive when the water activity is below 0.60 , and the growth of the most bacteria, yeast, and fungi is inhibited when the water activity is below 0.85 [12]. The effect of dehydration on the water activity of thyme is shown in Figure 2. The water activity was reduced with the drying time. Meanwhile, a higher 
drying temperature accelerated the decrease of the water activity. Firstly, the water activity decreased slowly to about 0.9 , and then decreased quickly to about 0.4 . Remarkably, the water activity of 0.60 has been regard as a better criterion of the dehydrated cabbage [8]. Based on the Figure 1 and Figure 2, the water activity of 0.60 of the dehydrated thyme was achieved early than the moisture content of $7 \%$ for each drying temperature. Hence, the moisture content of $7 \%$ guaranteed the safety of the dehydrated thyme.

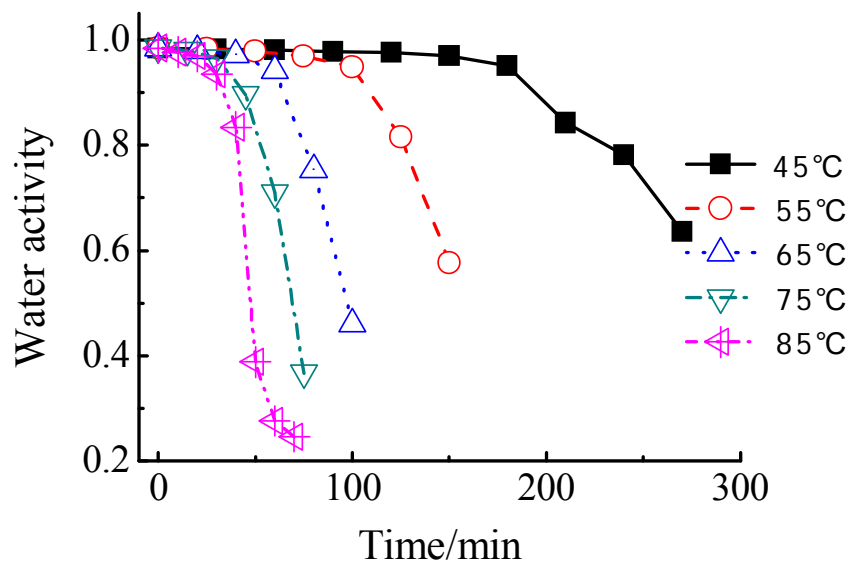

Figure 2 Effect of dehydration on water activity of thyme

Effect of dehydration on chlorophyll content of thyme. Chlorophylls are susceptible to physical, chemical or enzymatic degradation reactions [13]. The simultaneous actions of enzymes, oxygen, light and heat lead to the conversation from chlorophyll $\rightarrow$ pheophytin $\rightarrow$ pheophorbide. The rapid conversion of chlorophyll to pheophytin suggests rapid acidic removal of $\mathrm{Mg}^{2+}$ upon addition of dressing, whereas the rather slow conversion of pheophytin to pheophorbide suggests an enzymatic reaction that is mediated chlorophyllase [14]. The effect of drying temperature on the chlorophyll content of thyme is shown in Figure 3. The chlorophyll content decreased with the drying time. Meanwhile, a higher temperature accelerated the degradation of the chlorophyll in the thyme. The chlorophyll degradation of cabbages [15] and spinach [16] has been proved to follow the first-order-kinetics reaction. The degradation rate was highly exponential with temperature, thereby the degradation rate at a low temperature was much lower than that at a high temperature. A lower temperature was more effective to hold the chlorophyll content of the thyme.

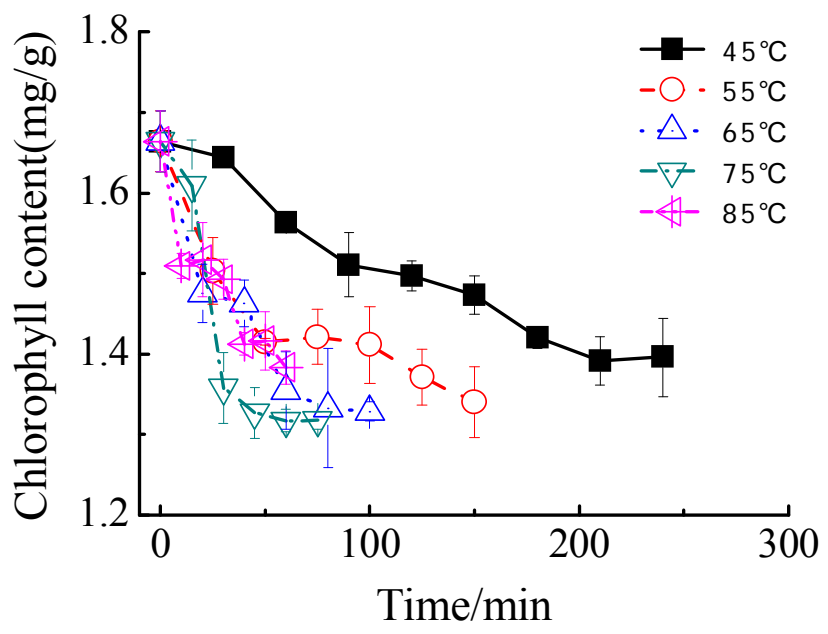

Figure 3 Effect of dehydration on chlorophyll content of thyme 


\section{Conclusions}

The moisture content and water activity of the thyme decreased with the drying time and drying temperature. The chlorophyll content was enhanced when the drying temperature was low. Hence, a lower drying temperature was better to hold the quality of thyme.

\section{Acknowledgements}

The authors are grateful to financial support of the earmarked fund for Modern Agro-industry Technology Research System (CARS-26-22 \& CARS-25), Beijing Academy of Agricultural and Forestry Sciences, New Discipline Breeding (KJCX20140204), and Beijing Key Laboratory of Fruits and Vegetable Storage and Processing (Z141105004414037).

\section{References}

[1] R. Morales, Taylor \& Francis, London 2002.

[2] H. Morais, C. Ramos, E. Forgacs, T. Cserhati, J. Oliviera: J. Chromatograp. B: Anal. Technol. Biomed. Life Sci. Vol 770 (2002) 297.

[3] B. Nickavar, F. Mojab, R. Dolat-Abadi: Food Chem. Vol 90 (2005) 609.

[4] M. Rahimmalek, S. A. H. Goli: Indust. Crops Prod. Vol 42 (2013) 613.

[5] I. Hamrouni-Sellami, I. Bettaieb Rebey, J. Sriti, F. Zohra Rahali, F. Limam, B. Marzouk: Food Bioprocess Technol. Vol 5 (2012) 2978.

[6] C. M. Díaz-Maroto, S. M. Pérez-Coello, A. M. González Vinas, D. M. Cabezudo: J. Agric. Food Chem. Vol 51 (2003) 1265.

[7] S. Sarosi, L. Sipos, Z. Kokai, Z. Pluh 谩 r, B. Szilvassy, I. Novak: Indust. Crops Prod. Vol 46 210.

[8] D. Wang, L. Jiang, X. Zhao, C. Zhang, Y. Ma: Adv. Mater. Res. Vol 396-398 (2012) 1297.

[9] M. A. Goula, G. K. Adamopoulos: Drying Technol. Vol 28 (2010) 752.

[10] M. C. Díaz-Maroto, M. S. Pérez-Coello, M. A. G. Viñas, M. D. Cabezudo: J. Agric. Food Chem. Vol 51 (2003) 1265.

[11] S. S. Sablani, S. Kasapis, M. S. Rahman: J. Food Eng. Vol 78 (2007) 266.

[12]V. Bohm, N. L. Putpitasarı-Nienaber, M. G. Ferruzzi, S. J. Schwartz: Food Chem. Vol 50 (2002) 221.

[13] N. Koca, F. Karadeniz, H. S. Burdurlu: Food Chem. Vol 100 (2006) 609.

[14] W. J. Heaton, Y. R. Yada, G. A. Marangoni: J. Agric. Food Chem. Vol 44 (1996) 395.

[15] D. M. Wang, L. Z. Jiang, X. Y. Zhao, C. Zhang, Y. Ma: Adv. Mater. Res. Vol 396-398 (2011) 1297.

[16] F. M. Lajolo, U. M. Lanfer Marquez: J. Food Sci. Vol 47 (1982) 1995. 